\title{
The Current and Evolving Therapeutic Paradigm in the Management of Metastatic Renal Cell Carcinoma
}

\author{
Thomas Hutson, MD ${ }^{1}$, Robert A. Figlin, MD, FACP ${ }^{2}$ \\ ${ }^{1}$ Charles A. Sammons Cancer Center, Texas A\&M College of Medicine, Dallas, TX \\ ${ }^{2}$ Cedars-Sinai Samuel Oschin Comprehensive Cancer Institute, Cedars-Sinai Health System, Los Angeles, CA
}

https://doi.org/10.52733/kcj19n3-r1

There has been tremendous progress in the treatment landscape of advanced or metastatic RCC since the advent of efficacious targeted therapies, immunotherapies and combinatorial regimens, leading to rapid expansion of therapeutic armamentarium over the last two decades. New advances have offered considerable improvement in prognosis, treatment-related toxicities, quality of life, and survival for patients with mRCC. Despite such advantages, there is an unmet need for developing novel biomarkers predictive of treatment response, optimizing treatment selection, and also improving strategies to overcome therapeutic resistance in heterogenous RCC tumors. Herein, we outline currently available first- and later-lines treatment strategies, unprecedented changes, and also discuss challenges in the treatment landscape of RCC.

\section{INTRODUCTION}

$\mathrm{R}$ enal cell carcinoma (RCC) remains one of the top ten most frequently diagnosed neoplasms with an incidence of over 403,000 new cases and 175,000 deaths globally. ${ }^{1}$ In the United States alone, about 73,750 new cases of kidney cancer were diagnosed, accounting for an estimated 14,830 deaths. In patients with RCC, about $30 \%$ of patients present with metastatic disease at the time of initial diagnosis typically requiring systemic therapy. Almost 30\% of patients who are treated for localized RCC develop a recurrent disease during the follow-up and the 5-year survival rate remains $12 \%$ in patients with mRCC. ${ }^{2}$ Given that RCC is chemo-resistant and radiation-resistant, and only a minority of patients with metastatic RCC surviving past 5 years, the treatment for the late-stage recurrent metastatic RCC remains highly challenging.

The precise treatment selection for patients with advanced or metastatic ccRCC has been guided by risk stratification models during the initial evaluation of patients. The Memorial Sloan Kettering Cancer Center (MSKCC) and the International Metastatic Renal Cell Carcinoma Database Consortium
(IMDC) are the most used nomograms and both frameworks have significant prognostic implications. Initially developed in the era of cytokine therapy, the MSKCC nomogram-based risk stratification has been updated for upfront decision-making in the current era of targeted therapies and immunotherapy. A modified version of the MSKCC nomogram was developed for decision-making in patients with previously treated RCC. ${ }^{3}$ Per MSKCC guidelines, patients are categorized into risk groups based on five criteria: good-risk (o risk factors), intermediate-risk (1-2), or poor-risk $(\geq 3)$. The IMDC or Heng criteria wer developed in the era of targeted therapies and it overlaps with the MSKCC model. IMDC also classifies patients into three risk groups. Favorable risk (o factors) with a median OS of 43.2 months, intermediate risk (1-2 factors) with a median OS of 22.5 months, and poor risk (3+ factors) with a median OS of 7.8 months. Despite the widespread utility of the IMDC model as a risk stratification tool for clinical trials involving VEGFR TKI and combination regimens, its applicability to IO therapies is rather limited. Although not widely used, other clinical practice guidelines have been issued by the National Comprehensive Cancer Network (NCCN), the European Society for Medical Oncology (ESMO), the European Organization for Research and Treatment of Cancer (EORTC), and the European Association of Urology (EAU).

\section{FIRST-LINE THERAPIES}

Given the large armamentarium of targeted therapies available alone or in combination, survival benefit is prolonged, and tolerability is enhanced for patients with metastatic clear cell renal cell carcinoma over almost two decades. And yet, physicians currently have to go through the difficult task of choosing the most optimal therapeutic regimen for first-line therapy. In the rapidly evolving therapeutic landscape of mRCC, this complexity is clear owing to enormous treatment options and access to new drugs arising from the latest clinical trials that lack real-world evidence. Especially, patient characteristics and survival outcomes in randomized trials may not accurately reflect a real-life clinical practice scenario. For the preferred treatment selection, IMDC risk stratification and PD-L1 biomarker status may provide some guidance. Treatment selection depends on several factors that include the patient's performance status, comorbidities, cancer subsets, and extent of disease burden as well as non-clinical factors namely, cost-effectiveness, and institutional availability, etc. Therefore, standardizing algorithms for optimized treatment sequencing remains a challenge. In recent years, the first-line treatment landscape has transitioned from recombinant cytokines to tyrosine kinase inhibitors (TKI), mammalian target of rapamycin (mTOR) inhibitors, and most recently, the immune checkpoint inhibitors (ICI). ${ }^{3}$ In this review, we highlight some of the recent and ongoing trials of ICI, ICI/TKI combinations, and novel HIF-2 $\alpha$ inhibitor agents that may potentially prolong survival benefits in patients with advanced and metastatic RCC. 

AVOREN trial of bevacizumab, a monoclonal antibody directed against the VEGF

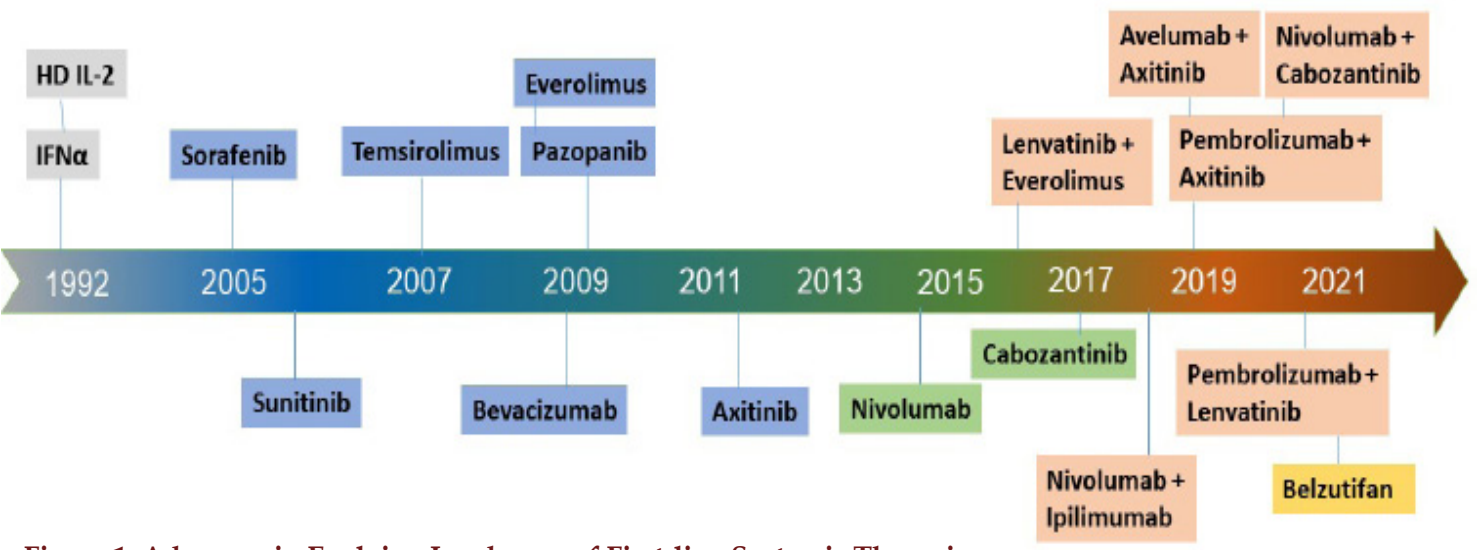
receptor (VEGFR) plus interferon- $\alpha-2 a$ (IFN a-2a) showed significant improvements in PFS (10.2 vs. 5.4 months, p $=0.0001)$ in contrast to treatment with interferon- $\alpha$ monotherapy in mRCC. ${ }^{10}$ Median OS was 23.3 months with bevacizumab plus IFN and 21.3 months with IFN plus placebo. Although bevacizumab-IFN showed OS benefit, its use was for Metastatic Renal Cell Carcinoma

\section{First-line VEGF- Targeted Therapies}

Before the advent of targeted therapies, cytokine-based traditional therapies i.e. interferon-alpha (IFN- $\alpha$ ) or interleukin-2 had been the mainstay of the RCC landscape until 2005. These therapies provided only a modest survival in the majority of patients but also resulted in a substantial incidence of high-grade adverse events. ${ }^{4}, 5$ With the improved understanding of implications of von Hippel-Lindau (VHL) gene mutations in angiogenic pathways, many VEGFbased TKIs were developed and eventually they evolved as defacto choice of first-line systemic therapy since $2005{ }^{6}$ Currently approved VEGF-targeted therapies either selectively inhibit VEGF receptors (eg, sorafenib, sunitinib, pazopanib, axitinib), or target circulating VEGF ligands (eg, bevacizumab), and block tumor angiogenesis. Whereas agents like everolimus and temsirolimus agents exert cytotoxic effects by inhibiting mTORC1, blocking protein synthesis, and cell-cycle progression.

For more than a decade, sunitinib, an orally administered multitargeted TKIs remained as the standardof-care and as the main comparator arm to clinical trials in first-line mRCC treatment. The survival benefit of sunitinib was evident in the pivotal randomized phase III trial in which sunitinib treatment resulted in improved PFS as compared with interferon in the first-line setting (11.0 vs. 5.0 months; $p<0.001$ ).7 Although a higher OS in patients treated with sunitinib was observed compared with those treated with IFN- $\alpha$ (26.4 versus 21.8 months, respectively), it lacked statistical significance. ${ }^{7}$ Based on the outcome, sunitinib was approved multinationally for the first- and second-line treatment of metastatic renal cell carcinoma (mRCC).

Here we will discuss results from important clinical trials involving VEGF targeting agents such as sorafenib, pazopanib, bevacizumab, and tivozanib as first-line therapy. A phase 3, randomized, double-blind, placebo-controlled study (TARGET) showed that treatment with sorafenib, a multikinase inhibitor results in improved progression-free survival (PFS) versus placebo in the second-line setting after cytokine therapy (5.5 vs. 2.8 months; $\mathrm{P}<0.01)^{8}$ Sorafenib therapy improved progression-free survival (PFS) in patients with advanced clear-cell renal-cell carcinoma in whom previous therapy has failed. However, sorafenib treatment is associated with increased toxic effects; rare serious adverse events such as hypertension and cardiac ischemia were more common in patients receiving sorafenib than in those receiving placebo. ${ }^{8}$ In other international, phase 3 study (COMPARZ), pazopanib and sunitinib therapy had comparable efficacy as compared with placebo or interferon. Pazopanib was non-inferior to sunitinib regarding PFS with similar OS between two arms. However, in terms of the patients perspective based on PISCES trial, more treatment-naive patients preferred pazopanib over sunitinib due to quality of life and safety. ${ }^{9}$ In the randomized phase III study (VEG105192; NCTo0334282), pazopanib demonstrated statistically and clinically meaningful improvement of PFS versus placebo (9.2 vs. 4.2 months; $p<0.0001$ ) in patients who had progressed after cytokines. Based on this data, the FDA approved both pazopanib and bevacizumab in combination with interferon limited by the toxicity of the regimen..$^{10}$ Overall, this AVOREN trial confirmed that bevacizumab plus IFN remains the first-line standard of care for patients with mRCC.

Similarly, a randomized controlled TIVO-1 trial has shown that tivozanib, a potent VEGFR-1, VEGFR-2, and VEGFR-3 inhibitor prolongs PFS (12.7 months) as compared with sorafenib (9.1 months) in the prespecified subpopulation of treatment-naive patients. ${ }^{11}$ Similarly, cabozantinib is an oral TKI that targets multiple tyrosine kinases, including hepatocyte growth factor (cMet), VEGFRs, and AXL. In CABOSUN trial, cabozantinib therapy improved PFS (8.2 vs. 5.6 months) and ORR ( $46 \%$ vs. $18 \%$ ) and reduced the rate of progression or death as compared to sunitinib in treatment-naïve patients with intermediate and poor IMDC risk group. ${ }^{12}$ Currently, cabozantinib represents a suitable targeted first-line agent especially among patients who are not eligible to receive immunotherapy.

\section{Front-Line mTOR Inhibitor Therapy}

In parallel to the development of VEGF inhibitors, mTOR inhibitors were also evolved in the mRCC landscape. Temsirolimus, a potent mTOR inhibitor, was approved for the treatment of advanced RCC after the multicenter, phase 3 ARCC trial (NCTooo65468). In this, treatment with temsirolimus has improved OS compared with interferon (10.9 vs. 7.3 months; $p=.008$ ) in treatment-naïve and poor risk mRCC patients. Temsirolimus monotherapy yielded longer OS (HR for death, $0.73 ; 95 \% \mathrm{CI}, 0.58$ to $0.92 ; \mathrm{P}=0.008$ ) and PFS $(\mathrm{P}<0.001)$ as compared to 


\begin{tabular}{|c|c|c|c|c|c|c|c|}
\hline \multirow{2}{*}{ Study RCT } & \multirow{2}{*}{ Treatment arms } & \multicolumn{2}{|c|}{ ORR } & \multicolumn{2}{|r|}{ PFS } & \multicolumn{2}{|c|}{ OS } \\
\hline & & ORR\% (CR) & HR $(95 \% \mathrm{Cl})$ & Median, mo & HR $(95 \% \mathrm{Cl})$ & Median, mo & HR $(95 \% \mathrm{Cl})$ \\
\hline \multirow{5}{*}{ Checkmate 214} & $\begin{array}{l}\text { Int/Poor risk } \\
\text { NIVO/IPI }(N=425) \\
\text { SUN }(N=422)\end{array}$ & $\begin{array}{l}42.1 \%(10.1) \\
26.3 \%(1.4)\end{array}$ & $P<0.001$ & $\begin{array}{l}11.6 \\
8.3\end{array}$ & $\begin{array}{l}0.75(0.62-0.90) \\
(P=0.015)\end{array}$ & $\begin{array}{l}47 \\
26.6\end{array}$ & $\begin{array}{l}0.66(0.55-0.80) \\
(P<0.0001)\end{array}$ \\
\hline & $\begin{array}{l}\text { Favorable risk } \\
\text { NIVO/IPI }(N=125) \\
\text { SUN }(N=124)\end{array}$ & $\begin{array}{l}28.8 \%(12.8) \\
54.0 \%(5.6)\end{array}$ & $P<0.0001$ & $\begin{array}{l}17.0 \\
28.8\end{array}$ & $\begin{array}{l}1.65(1.16-2.35) \\
(P=0.0049)\end{array}$ & $\begin{array}{l}\text { NR } \\
\text { NR }\end{array}$ & $\begin{array}{l}1.19(0.77-1.85) \\
(P=0.43)\end{array}$ \\
\hline & $\begin{array}{l}\text { ITT } \\
\text { NIVO/IPI }(N=550) \\
\text { SUN }(N=546)\end{array}$ & $\begin{array}{l}39.1 \%(10.7) \\
32.6 \%(2.4)\end{array}$ & $P=0.02$ & $\begin{array}{l}12.4 \\
12.3\end{array}$ & $\begin{array}{l}0.88(0.75-1.04) \\
(P=0.126)\end{array}$ & $\begin{array}{l}\text { NR } \\
38.4\end{array}$ & $\begin{array}{l}0.72(0.49-0.95) \\
(P=0.0002)\end{array}$ \\
\hline & $\begin{array}{l}\text { Int/Poor }(P D-L<1) \\
\text { NIVO/IPI }(N=284) \\
\text { SUN }(N=278)\end{array}$ & $\begin{array}{l}37 \% \\
28 \%\end{array}$ & $P=0.03$ & $\begin{array}{l}11.0 \\
10.4\end{array}$ & $\begin{array}{l}1.00 \\
(0.8-1.26)\end{array}$ & $\begin{array}{l}\text { NR } \\
\text { NR }\end{array}$ & $0.73(0.56-0.96)$ \\
\hline & $\begin{array}{l}\text { Int/Poor (PD-L > 1) } \\
\text { NIVO/IPI }(N=100) \\
\text { SUN }(N=114)\end{array}$ & $\begin{array}{l}58 \% \\
22 \%\end{array}$ & $P<0.001$ & $\begin{array}{l}22.8 \\
5.9\end{array}$ & $\begin{array}{l}0.46 \\
(0.31-0.67)\end{array}$ & $\begin{array}{l}\text { NR } \\
\text { NR }\end{array}$ & $0.45(0.29-0.71)$ \\
\hline \multirow{3}{*}{ KEYNOTE 426} & $\begin{array}{l}\text { ITT } \\
\text { AXI/PEMBRO }(N=432) \\
\text { SUN }(N=429)\end{array}$ & $\begin{array}{l}60 \%(9) \\
40 \%(3)\end{array}$ & $P<0.0001$ & $\begin{array}{l}15.4 \\
11.1\end{array}$ & $\begin{array}{l}0.71(0.60-0.84) \\
P<0.0001\end{array}$ & $\begin{array}{l}\text { NR } \\
35.7\end{array}$ & $\begin{array}{l}0.68(0.55-0.85) \\
P=0.0003\end{array}$ \\
\hline & $\begin{array}{l}\text { Int/Poor risk } \\
\text { AXI/PEMBRO }(N=294) \\
\text { SUN }(N=298)\end{array}$ & $\begin{array}{l}55.8 \%(8) \\
35.2 \%\end{array}$ & - & $\begin{array}{l}12.7 \\
8.3\end{array}$ & $\begin{array}{l}0.69(0.56-0.84) \\
P=0.0002\end{array}$ & $\begin{array}{l}\text { NR } \\
28.9\end{array}$ & $\begin{array}{l}0.63(0.50-0.81) \\
P=0.0001\end{array}$ \\
\hline & $\begin{array}{l}\text { Favorable Risk } \\
\text { AXI/PEMBRO }(N=138) \\
\text { SUN }(N=131)\end{array}$ & $\begin{array}{l}69.6 \%(11) \\
50.4 \%(6)\end{array}$ & - & $\begin{array}{l}20.8 \\
18.0\end{array}$ & $\begin{array}{l}0.79(0.57-1.09) \\
P=0.078\end{array}$ & $\begin{array}{l}\text { NR } \\
\text { NR }\end{array}$ & $\begin{array}{l}1.06(0.6-1.86) \\
P=0.58\end{array}$ \\
\hline \multirow{3}{*}{ IMmotion 151} & $\begin{array}{l}\text { PD-L1+ } \\
\text { ATEZO/BEV }(N=178) \\
\text { SUN }(N=184)\end{array}$ & $\begin{array}{l}\text { Inv } \\
43 \%(9) \\
35 \%(4)\end{array}$ & - & $\begin{array}{l}\text { Inv } \\
11.2 \\
7.7\end{array}$ & $\begin{array}{l}0.74(0.57-096) \\
P=0.02\end{array}$ & $\begin{array}{l}34.0 \\
32.7\end{array}$ & $\begin{array}{l}0.84(0.62-1.15) \\
P=0.28\end{array}$ \\
\hline & $\begin{array}{l}\text { PD-L1 + } \\
\text { ATEZO/BEV }(N=178) \\
\operatorname{SUN}(N=184)\end{array}$ & $\begin{array}{l}\text { IRC } \\
36 \%(15) \\
33 \%(8)\end{array}$ & - & $\begin{array}{l}\text { IRC } \\
8.9 \\
7.2\end{array}$ & $\begin{array}{l}0.93 \\
(0.72-1.21)\end{array}$ & - & - \\
\hline & $\begin{array}{l}\text { ITT } \\
\text { ATEZO/BEV }(N=454) \\
\operatorname{SUN}(N=461)\end{array}$ & $\begin{array}{l}\text { Inv } \\
37 \%(5) \\
33 \%(2)\end{array}$ & - & $\begin{array}{l}\text { Inv } \\
11.2 \\
8.4\end{array}$ & $\begin{array}{l}0.83(0.70-0.97) \\
P=0.02\end{array}$ & $\begin{array}{l}33.4 \\
34.9\end{array}$ & $\begin{array}{l}0.93(0.76-1.14) \\
P=0.47\end{array}$ \\
\hline \multirow{2}{*}{ JAVELIN Renal 101} & $\begin{array}{l}\text { PD-L1 + } \\
\text { AXI/AVEL }(N=270) \\
\text { SUN }(N=290)\end{array}$ & $\begin{array}{l}\text { BICR } \\
55.9 \%(5.6) \\
27.2 \%(2.4)\end{array}$ & $\begin{array}{l}\mathrm{OR}=3.389 \\
(2.34-4.90)\end{array}$ & $\begin{array}{l}\text { BICR } \\
13.8 \\
7.0\end{array}$ & $\begin{array}{l}0.62(0.49-0.77) \\
P<0.0001\end{array}$ & $\begin{array}{l}\text { NR } \\
28.6\end{array}$ & $\begin{array}{l}0.83(0.59-1.15) \\
P=0.13\end{array}$ \\
\hline & $\begin{array}{l}\text { ITT } \\
\text { AXI/AVEL }(N=442) \\
\text { SUN }(N=444)\end{array}$ & $\begin{array}{l}\text { BICR } \\
52.5 \%(3.8) \\
27.3 \%(2.0)\end{array}$ & $\begin{array}{l}\mathrm{OR}=2.99 \\
(2.23-3.99)\end{array}$ & $\begin{array}{l}\text { BICR } \\
13.8 \\
8.4\end{array}$ & $\begin{array}{l}0.69(0.57-0.82) \\
P<0.0001\end{array}$ & $\begin{array}{l}\text { NR } \\
\text { NR }\end{array}$ & $\begin{array}{l}0.80(0.61-1.02) \\
P=0.039\end{array}$ \\
\hline \multirow[b]{2}{*}{ CLEAR } & $\begin{array}{l}\text { ITT } \\
\text { LENV/PEMBRO }(N=454)\end{array}$ & $71 \%(16)$ & $P<0.001$ & 23.9 & $\begin{array}{l}0.39(0.32-0.49) \\
P<0.001\end{array}$ & $\mathrm{NR}^{\mathrm{b}}$ & $\begin{array}{l}0.66(0.49-0.88) \\
P=0.005\end{array}$ \\
\hline & LENV/EVERO $(N=461)$ & $53 \%(10)$ & $P<0.001$ & 14.7 & $\begin{array}{l}0.65(0.53-0.80) \\
P<0.001\end{array}$ & $\mathrm{NR}^{\mathrm{b}}$ & $\begin{array}{l}1.15(0.88-1.5) \\
P=0.30\end{array}$ \\
\hline Checkmate 9ER & $\begin{array}{l}\text { ITT } \\
\text { CABO/NIVO }(N=323) \\
\operatorname{SUN}(N=328)\end{array}$ & $\begin{array}{l}55.7 \%(8) \\
27.1 \%(5)\end{array}$ & $P<0.0001$ & $\begin{array}{l}16.6 \\
8.3\end{array}$ & $\begin{array}{l}0.51(0.41-0.64) \\
P<0.0001\end{array}$ & $\begin{array}{l}\text { NR } \\
\text { NR }\end{array}$ & $\begin{array}{l}0.60(0.40-89) \\
P=0.001\end{array}$ \\
\hline
\end{tabular}

Table 1. Summary of phase III front-line combination trials in Renal Cell Carcinoma. Abbreviations: ATEZO: ATEZOlizumab; AVEL: AVELumab; AXI: AXItinib; BEV: BEVacizumab; CABO: CABOzantinib; CI: confidence interval; EVERO: EVEROlimus; HR: hazard ratio; IMDC: International Metastatic Renal Cell Carcinoma; int: intermediate; inv: investigator; IPI: IPllimumab; IRC: independent review committee; ITT: intention to treat; LENV: LENVatinib; mo: month(s); NA: not applicable; NIVO: NIVOlumab; NR: not reached; ORR: objective response rate; OS: overall survival; PD-L1: programmed cell death ligand 1; PFS: progression free survival; Ph: phase; q3wk: every 3 weeks; qd: once daily; r: randomized; Sun: sunitinib; wk: week.

interferon alone ${ }^{13}$ However, the combination of interferon with temsirolimus also did not improve PFS or OS and only a modest improvement regarding PFS versus interferon (5.5 vs. 3.1 months) was observed. ${ }^{13}$ Based on this, temsirolimus was approved by the FDA for the first-line treatment of advanced RCC in May 2007. A more pronounced survival advantage was observed only in patients with non-clear cell histology. However, the RECORD-3 trial subsequently showed everolimus was inferior to sunitinib across IMDC risk groups. Given such modest results and also due to its weekly intravenous injection limitation, temsirolimus is not a widely used therapy in front-line for patients and its utility has been relegated to second or later lines of therapy with mRCC who have poor risk prognostic features. Another mTOR inhibitor everolimus has been evaluated in ESPN study for the first-line setting in patients with metastatic nccRCC. In this, everolimus failed to yield a survival advantage as sunitinib had better mPFS than that of everolimus (6.1 months vs, 4.1 months, $p=0.25) .{ }^{14}$ Therefore, everolimus is not recommended in the first-line treatment for nccRCC.

\section{ICI Based First-Line Therapies}

Owing to their robust and clinically relevant survival benefits, immune checkpoint-inhibitor (ICI) proteins including anti-programmed death receptor 1 (PD1), anti-programmed death receptor ligand 1 (PD-L1), and anti-cytotoxic $\mathrm{T}$ lymphocytes antigen-4 (CTLA-4) have been integrated into the therapeutic landscape as the first-line as well as second-line treatment for moderate to high-risk mRCC. ${ }^{3}$ For instance, since the approval of the CTLA- 4 antibody ipilimumab in patients with melanoma in 2011, the footprints of ICIs also expanded across the RCC landscape following studies of several PD-1/PD-L1 inhibitors including nivolumab, pembrolizumab, atezolizumab, durvalumab, and avelumab as well as the CTLA-4 inhibitor ipilimumab. Similarly, nivolumab a humanized IgG4 anti-PD-1, was the first 
ICI in kidney cancer space approved by FDA in 2015. Such developments revolutionized the ICI-based immunotherapies in patients with refractory mRCC.

CheckMate 214 (NCTo2231749) is the first trial in the RCC landscape to evaluate the CTLA-4 blocker (ipilimumab) and PD-1 inhibitor (nivolumab) combination in the IMDC intermediate or the high-risk population. ${ }^{15}$ The results validated the proof of concept that PD-1 inhibitor plus CTLA-4 blocker combination can deliver synergistic benefit as compared to the anti-VEGF TKI sunitinib in the first-line metastatic RCC setting. Improved response rates $(42 \%, 9 \% \mathrm{CR}$ vs $27 \%, 1 \% \mathrm{CR}$; $\mathrm{p}<0.001$ ), PFS (11.6 mo vs 8.4 mo, HR $0.82, \mathrm{p}=0.03$ ) and OS (NR vs $26.6 \mathrm{mo}$; HR 0.66; 95\% CI 0.54-0.80; $\mathrm{p}<0.0001$ ) were observed in combination arm as compared to sunitinib. In particular, the addition of ipilimumab to nivolumab resulted in significantly better overall survival and improved ORR as compared to sunitinib, secured a place in the firstline treatment algorithm. ${ }^{15}$ In phase III, randomized keynote-426 trial, treatment with pembrolizumab plus axitinib resulted in significantly longer OS and PFS, as well as a higher ORR, than treatment with sunitinib among patients with previously untreated advanced renal-cell carcinoma. ${ }^{16}$ Pembrolizumab plus axitinib therapy resulted in better OS (median not reached) as compared to therapy with sunitinib (35.7 months; HR $0.68, p=0.0003$ ) and higher PFS (median 15.4 months vs 11.1 months, HR 0.69; $\mathrm{p}<0.0001)$. As compared to sunitinib arm, the combination arm also had better CR ( $9 \%$ versus $3 \%$ ) and ORR $(59.3 \%$ vs $35.7 \%, \quad \mathrm{p}<0 \cdot 0001){ }^{16}$ This study validated the benefit of pembrolizumab + axitinib combination therapy. Similarly, pembrolizumab monotherapy for treatment naïve patients has also demonstrated promising efficacy and acceptable tolerability in patients with accRCC in the KEYNOTE-427 (NCTo2853344) trial.

\section{HIF inhibitor based therapies}

Belzutifan, a highly selective hypoxia-inducible factor inhibitor (HIF-2 $\alpha$ ), offers a novel approach, taking a different path than commonly used, to treat RCC. Most recently, the open-label study 004 (NCT03401788) has evaluated the efficacy of belzutifan in patients with VHL-associated RCC. ${ }^{17}$ In patients with VHL-associated RCC $(\mathrm{n}=61)$, belzutifan resulted in an ORR of 49\% (95\% CI, 36\%-62\%); all responses were partial responses. Median DOR had not yet been reached (range, $2.8+$ to
22.3+ months); among responders, 56\% $(n=17 / 30)$ were still responding after at least 12 months. Median TTR was eight months (range, 2.7-19 months). Based on these data, FDA approved belzutifan for adult patients with VHL disease who require therapy for RCC and other tumors. ${ }^{17}$ Currently belzutifan was investigated in phase III trials as part of combination first-and second-line therapies for advanced clear cell renal cell carcinoma and also as monotherapy for previously treated patients.

\section{Combinatorial Therapies: ICI Plus TKI}

Tumor angiogenesis is largely driven by VEGF-mediated mechanisms in kidney cancers. Apart from exerting a proangiogenic effect, VEGFs also mediate immunosuppression in the tumor microenvironment. Interestingly, by promoting the accumulation of myeloid-derived suppressor cells and regulatory $\mathrm{T}$ cells and also by impeding the migration of $\mathrm{T}$ lymphocytes towards the tumor microenvironment, VEGFs potentially drive angiogenesis. Disruption of VEFG has been shown to facilitate promote cytotoxic T-cell infiltration into the tumor microenvironment and also decrease the activity of T-regulatory cells and myeloid-derived suppressor cells, thereby enhancing responsiveness to immunotherapy. ${ }^{18}$ Such synergistic effectiveness of ICI agents in combination with either multi-kinase inhibitors or other monoclonal antibodies (CTLA4 and PD-1) have gained momentum and have been rapidly integrated into the first line treatment landscape.

There are several ongoing clinical studies based on doublet and triplet regimens for treatment-naïve metastatic or advanced ccRCC. In a phase II, randomized study (IMmotion150) by $\mathrm{McDermott}$ et al. investigated the combination of atezolizumab, an anti-PD-L1 antibody, with bevacizumab, an anti-VEGF agent as compared to sunitinib monotherapy in mRCC19. In ITT patient population, improved median PFS was noted with atezolizumab plus bevacizumab combination group (11.7 vs. 8.4 months).19 Besides, in PDL1 positive patients, the combination arm had a PFS of 14.7 months versus 7.8 months with sunitinib. The ORR in PD-L1+ patients was $43 \%$ in the combination arm as compared to $35 \%$ in the sunitinib arm. The CR rate in the PD$\mathrm{L} 1+$ patients was $9 \%$ in the combination arm as compared to $4 \%$ in the sunitinib arm. ${ }^{19}$ In a phase III IMmotion 151 trial (NCTo2420821), similar survival benefits were obtained. Similarly, CheckMate
016, a phase I, open-label, parallel-cohort, dose-escalation study investigated the efficacy and safety of nivolumab plus ipilimumab in combination, and nivolumab plus a TKI. ORR was 40.4\% in both arms, with ongoing responses in $42.1 \%$ and $36.8 \%$ of patients in the N3I1 and N1I3 arms, respectively. This combination demonstrated manageable safety, notable antitumor activity, and durable responses with promising OS in patients with mRCC progressed after prior therapy. In another clinical trial (NCTo3075423), a combination of pembrolizumab plus axitinib resulted in significantly longer OS and PFS, as well as a higher ORR, than treatment with sunitinib16. After a median follow-up of 12.8 months, the estimated percentage of patients who were alive at 12 months was $89.9 \%$ in the pembrolizumab-axitinib group and $78.3 \%$ in the sunitinib group. Median PFS was 15.1 months in the pembrolizumab-axitinib group and 11.1 months in the sunitinib group. The objective response rate was $59.3 \%$ in the pembrolizumab-axitinib group and $35.7 \%$ in the sunitinib group $(\mathrm{P}<0.001) .{ }^{16}$

Similarly, pembrolizumab was also being evaluated in the KEYNOTE 427 phase II trial. Similarly, durvalumab is being evaluated in combination with savolitinib, a highly selective MET tyrosine kinase inhibitor, in the CALYPSO phase II trial (NCTO2819596). In another randomized phase III JAVELIN Renal 101 (NCT02684006) trial, Motzer et al investigated the combination of axitinib and avelumab in treatment-naive RCC patients with metastatic or advanced disease. ${ }^{20}$ Avelumab plus axitinib arm had longer PFS and a significantly higher objective response rate than those who received sunitinib monotherapy. Results showed that mPFS in the combination arm was 13.8 months versus 8.4 months in sunitinib arm (HR, 0.69; p<0.001), and the ORR and CR rate were $55 \%$ and $4 \%$ in the combination arm versus $26 \%$ and $2 \%$ in the sunitinib arm respectively.20 The safety profile was comparable to the results of the JAVELIN Renal 101 trial. The subgroup analysis indicates that the benefit of pembrolizumab plus axitinib for OS, PFS, and ORR was observed in the entire population irrespective of IMDC prognostic group and PD-L1 tumor expression.

In CheckMate-9ER study, nivolumab plus cabozantinib combination had significant benefits over sunitinib in terms of PFS, OS in patients with treatment naïve aRCC. The mPFS was 16.6 months with nivolumab plus 
cabozantinib and 8.3 months with sunitinib. ${ }^{12}$ The probability of OS at 12 months was $85.7 \%$ with the combination arm and $75.6 \%$ with sunitinib. An OR occurred in $55.7 \%$ of patients in the combination arm versus $27.1 \%$ in sunitinib arm $(\mathrm{P}<0.001)$. Efficacy benefits with nivolumab plus cabozantinib were consistent across subgroups. ${ }^{12}$ In another phase 3 trial, patients with aRCC and no previous systemic therapy were randomly assigned to receive lenvatinib plus pembrolizum$a b$, lenvatinib plus everolimus, or sunitinib. Lenvatinib plus pembrolizumab therapy resulted in longer PFS (median, 23.9 vs. 9.2 months) and OS than with sunitinib. However similar benefits were not observed with lenvatinib plus everolimus as compared to sunitinib. This study demonstrated lenvatinib plus pembrolizumab therapy was associated with significantly longer PFS and OS as compared to sunitinib. In a non-randomized Phase Ib/II study, VEGF-TKI plus IO (tivozanib plus nivolumab) combination was assessed in patients previously treated with one oral TKI (NCTo3136627). The ORR was $56 \%$, with one patient achieving a complete response. The combination of tivozanib with nivolumab prolonged disease control (median PFS of 18.9 months) and also showed a tolerable AE profile in both treatment-naïve and previously treated metastatic RCC. ${ }^{21}$

In phase III CLEAR clinical trial, lenvatinib plus pembrolizumab treatment significantly improved PFS compared to sunitinib (HR: 39, median = 23.9 vs 9.2 months). This combination also improved the ORR compared to sunitinib (71.0\% vs $36.1 \%)$ with an impressive complete response rate of $16.1 \%$ and OS was also significantly longer than sunitinib $(\mathrm{HR}=0.66)$. Although lenvatinib plus everolimus, in the third arm, significantly improved PFS compared to sunitinib (median $=14.7$ vs 9.2 months, $\mathrm{HR}=0.65,95 \% \mathrm{CI}=0.53-0.83)$, but overall survival benefit was inconclusive $(\mathrm{HR}=1.15)$. Based on the promising data, this combination became the fifth immuno-oncology combination for the first-line treatment of metastatic RCC, in addition to nivolumab plus ipilimum$\mathrm{ab}$, pembrolizumab plus axitinib, avelumab plus axitinib, and nivolumab plus cabozantinib.

Although the combination of ICI and antiangiogenics has shown encouraging preliminary antitumor activity for advanced or mRCC, high incidence of toxicity along with less favorable tolerability profile may compromise the benefits in patients. For instance, in the phase I study CheckMate 016 (NCTo1472081), the efficacy and safety of nivolumab in combination with antiangiogenic tyrosine kinase inhibitors or ipilimumab for the treatment of mRCC. ${ }^{22}$ In this study, the addition of sunitinib or pazopanib to nivolumab resulted in a high incidence of high-grade toxicities, limiting its scope in future trials. Given the possibility that long-term cumulative adverse effects from antiangiogenic and ICI-combinations may accumulate over time and outweigh the benefits, such combinatorial therapies warrant close monitoring to avoid unprecedented risks.

\section{VEGF-TKI plus mTOR inhibitors}

Targeted therapies directed towards both VEGFR and mTOR pathways have long been considered a potential synergistic strategy in mRCC landscape. However, only a few combinations shown a successful synergy with the benefits that outranged the combined toxicities from the regimens involved. Lenvatinib, a novel potent multi-target TKI of VEGFR 1-3, PDGFR- $\beta$, RET, c-KIT, and FGFR 1-4, was approved in combination with everolimus for the treatment of advanced RCC following one prior antiangiogenic therapy. In a phase 2 trial (NCTo1136733), 153 patients with mRCC previously treated with VEGF-TT were randomly allocated to receive either the combination of lenvatinib plus everolimus, single-agent lenvatinib, or single-agent everolimus. Lenvatinib plus everolimus significantly prolonged PFS compared with everolimus alone, but not compared with lenvatinib alone. ${ }^{23}$ This trial demonstrated that lenvatinib plus everolimus and lenvatinib alone resulted in a progression-free survival benefit for patients with metastatic renal cell carcinoma who have progressed after one previous VEGF-targeted therapy.

\section{VEGF inhibitor plus HIF inhibitor} The preliminary, interim results from a phase II trial has shown that belzutifan plus cabozantinib treatment resulted in $88 \%$ tumor shrinkage in target lesions and a disease control rate of $90 \%$ for patients with previously treated advanced clear cell RCC, according to preliminary results from cohort 2 in an ongoing phase II study. The PFS rate was $78 \%$ at 6 months and $65 \%$ at 12 months. Median progression-free survival was 16.8 months.

\section{SECOND-LINE THERAPIES}

Ideally, second-line therapies are designed to address the resistance mechanism obtained from first-line treatments and utilize different treatment modalities to gain better disease control in the second-line setting. For instance, for patients progressing despite first-line immunotherapy, TKIbased second-line therapy could deliver better outcome. If a patient has progressed on combination ICI/TKI treatment, the second-line regimen could include either a different TKI alone or in combination with an mTOR inhibitor. The different TKI is chosen based on their capacit still VEGF-TT

$y$ to challenge escape pathways that led to treatment resistance to the prior TKI, via AKT, MET, AXL, and FGF signaling. ${ }^{6}$ For patients progressing after firstline VEGF therapy, checkpoint inhibitors is not the only favorable therapy; TKI therapy can be also a viable option. Cabozantinib, an orally bioavailable TKI, inhibits VEGFRs, MET, and AXL targets implicated in the pathogenesis and progression of RCC. These favorable results in TKI-refractory disease support the hypothesis that the clinical activity of cabozantinib in RCC may result from combined inhibition of VEGFRs and additional targets, such as MET and AXL, that are not inhibited by other TKIs. ${ }^{8,10} 11$ Another added advantage is that the number of prior therapies does not seem to affect the efficacy of cabozantinib or nivolumab.

\section{Second-line post VEGFI therapy}

The following studies explored survival benefits in patients who progressed VEGFR TKI therapies. The international randomized, open-label, phase III METEOR trial assessed the efficacy and safety of cabozantinib with the mTOR inhibitor everolimus in patients with advanced RCC following one or more VEGFR TKI therapy. Treatment with cabozantinib improved PFS, ORR, and OS versus everolimus in patients with aRCC. In cabozantinib arm, median OS was 21.4 months (95\% CI 18.7-not estimable) as compared to $16 \cdot 5$ months in everolimus arm. Cabozantinib treatment also resulted in improved progression-free survival and objective response ( $17 \%$ with cabozantinib vs $3 \%$ with everolimus; $\mathrm{p}<0 \cdot 0001$ ). Cabozantinib treatment was also resulted in improved clinical outcomes in patients who had received both VEGFR TKI therapy and an immune checkpoint inhibitor. Cabozantinib may have added advantage over other therapies especially for patients with bone and brain metastasis subgroups that derived 
increased benefit, that is, elderly, good and intermediate-risk patients. In the METEOR trial, cabozantinib therapy resulted in better median PFS of 7.4 months and 5.6 months as compared to 2.7 months and 1.9 months with everolimus in those with bone metastasis and bone and visceral disease, respectively. The rate of post-randomization skeletal-related events was lower (16\%) with cabozantinib as compared to $34 \%$ events with everolimus. Taken together, these data strongly support the use of cabozantinib in mRCC patients with bone and brain metastasis.

The IO agent nivolumab can be given as a preferred second-line monotherapy option for patients who progressed on first-line anti-VEGF therapies. The CheckMate-025 (NCT01668784) trial has demonstrated that nivolumab delivers better PFS, overall response rate and overall survival, paving the way for the use of nivolumab as second-line therapy. ${ }^{24}$ In a randomized open-label, phase III study, a total of 821 advanced ccRCC patients who had received previous treatment with one or two regimens of antiangiogenic therapy were randomly assigned either nivolumab or everolimus. ${ }^{24}$ Results showed that ORR was greater with nivolumab than with everolimus ( $25 \%$ vs. $5 \%$; $<<0.001)$ and median PFS was better with nivolumab than with everolimus (4.6 months vs 4.4 months; $\mathrm{p}=0.11) .24$ Results indicated that the nivolumab arm had 25.0 months median OS as compared to 19.6 months in the everolimus arm. Nivolumab's OS benefit was evident across prespecified MSKCC risk and PD-L1 subgroups. Taken together, these results especially obtained from METEOR and CheckMate 025 trials highlight the broad clinical utility of cabozantinib or nivolumab as a preferred second-line therapy for previously treated patients with advanced RCC in the post VEGFI setting.

In a randomized, open-label trial, patients who received at least two previous systemic treatments (including at least one previous treatment with VEGFR inhibitor) were randomly assigned to receive tivozanib or sorafenib. ${ }^{25}$ Median PFS was significantly longer with tivozanib (5.6 months) than with sorafenib (3.9 months). This study showed that tivozanib as third-line or fourth-line therapy improved progression-free survival and was better tolerated compared with sorafenib in patients with metastatic renal cell carcinoma. In other randomized, phase 2, open-label, lenvatinib, everolimus, or their combination as a second-line treatment were assessed in patients with mRCC who had received treatment with a VEGFtargeted therapy. Lenvatinib plus everolimus significantly prolonged PFS compared with everolimus alone (median 14.6 months vs 5.5 months), but not compared with lenvatinib alone (7.4 months). Single-agent lenvatinib significantly prolonged PFS compared with everolimus alone. Lenvatinib plus everolimus and lenvatinib alone resulted in a PFS benefit for patients with mRCC who have progressed after one previous VEGF-targeted therapy.

\section{Second-line post IO therapy}

For those patients who progressed after initial ICI therapy, the second-line option would be VEGFRTKI. Currently, preferred second line choice was the inhibitor of the mammalian target of rapamycin pathway (mTOR) everolimus in 53.3\% of cases. Besides, alternative options including VEGFR-TKI options (cabozantinib) or mTOR combinations (lenvatinib plus everolimus) for a later rescue line are also considered. Similarly, RECORD-1 study shown demonstrated benefits from everolimus in patients who had received at least one prior treatment with sunitinib and/or sorafenib. The median PFS for everolimus was 4.0 vs 1.9 months with placebo. No OS impact was seen in the trial although the crossover rate was over $90 \%{ }^{26}$ For those who receive either pazopanib or sunitinib in the first-line therapy, a mammalian target of rapamycin (mTOR) inhibitor and a TKI could be a possible choice of second-line therapy. Another interesting question will be the possibility of ICI rechallenge and elucidating the use of cytoreductive nephrectomy within the context of new immunotherapeutic interventions.

For patient progressed on firstline ICI/TKI combination, a second-line combination mTOR inhibitors/TKIs could be another option available. Although single-agent temsirolimus versus sorafenib yielded a lack of benefit after progression on a VEGF-TKI in the second-line setting ${ }^{14}$, a randomized phase II Study 205 trial supported the use of lenvatinib plus everolimus vs lenvatinib alone vs everolimus alone in this setting. ${ }^{15} \mathrm{~A}$ superior PFS was noted at 14.6 months vs 5.5 months with everolimus alone, but it lacked a statistically significant difference when compared with the lenvatinib arm (PFS, 7.4 months). The study results led to the first FDA-approved combination of a TKI and an mTOR inhibitor. However, highgrade toxicities that occurred in the combination arm warrant newer-generation multi-TKI/mTOR such as vorolanib to identify better-tolerated regimens. ${ }^{16}$ Currently, a phase 1 trial involving vorolanib/everolimus combination in the second-line setting is underway.

Despite the robust profile of the anti-PD-1/anti-CTLA-4 dual checkpoint blockade, some mRCC patients do not respond well to the therapy and would have intermediate- and poor-risk IMDC factors. Given that the tumor is still VEGF-TT naïve in these subsets of patients, TKI approved for the first-line may have a role. For these patients, axitinib, cabozantinib, or the combination of lenvatinib + everolimus could be used as a second-line, depending on clinical and non-clinical factors. For patients who progressed after ICI therapy including ipilimumab/nivolumab, a phase 2 nonrandomized trial $(n=38)$ has shown that axitinib may provide clinical efficacy (PFS 9.2 mo, ORR 40\%). Besides, small retrospective studies that shown median PFS of 8 months using TKI after dual checkpoint blockade, supported the use of cabozantinib compared with sunitinib in this specific population. In a retrospective analysis involving 86 patients who received cabozantinib after progression on ICI alone, ICI plus VEGFIs or other therapies. Cabozantinib arm had ORR of $36 \%$ with no complete response and $43 \%$ achieving stable disease; $21 \%$ had primary progressive disease. The median OS was 13.1 months with OS rate of 12 months.

For patients who progressed on prior PD-1/PD-L1 or ICI/TKI therapy may benefit from the combination of lenvatinib and pembrolizumab. The phase II KEYNOTE-146 trial has evaluated the benefit of lenvatinib plus pembrolizum$\mathrm{ab}$ after up-front therapy with nivolumab/ipilimumab. ${ }^{12,27}$ The 12-month rate of PFS was $45 \%$, with a median value of 11.7 months, and the corresponding values for overall survival were $77 \%$ and not reached. The ORR was $55 \%$ for those given only PD-1 or PD-L1 inhibitors, and $59 \%$ and $47 \%$ for patients who had also received a VEGR inhibitor or nivolumab plus ipilimumab, respectively. ${ }^{27}$ For patients who progressing after either firstline IL-2 or temsirolimus, the preferred second-line therapy could be pazopanib or sunitinib. However, the treatment choice remains unclear for patients previously treated with ICI. In this setting, the preferred treatment choice could be 
VEGFR-TKI not previously used in combination with ICI therapy. For third-line therapy $63.5 \%$ of patients received TKI, primarily sorafenib and axitinib (23.5\% each), whereas $36.5 \%$ of patients received everolimus. For fourth-line and beyond, sorafenib was used in $21.2 \%$ of patients as fourth-line therapy, and both sunitinib and everolimus were used equally as fifth-line therapy, in $23.8 \%$ of patients. A pivotal phase III CONTACT-03 study (NCTo4338269) is currently underway in patients who received prior ICI as a first- or second-line treatment in RCC. For the patients who are ineligible to receive either nivolumab IO therapy or cabozantinib, axitinib is recognized as another appropriate second-line option according to the international guidelines and recommendations. Axitinib is recognized as another appropriate second-line option in this setting based on AXIS trial. In this, 23 patients with one prior therapy were randomized to axitinib or sorafenib, axitinib was established as the preferred second-line choice. ${ }^{28}$ The median PFS for axitinib was 8.3 vs 5.7 months with sorafenib, although no statistical difference in OS was observed.

\section{CONCLUDING REMARKS}

Over the last decade, there has been tremendous progress in the treatment landscape of mRCC with new and efficacious monotherapies and combinatorial regimen, leading to an expansion of therapeutic armamentarium. With the continuous implementation of several first-line therapies (eg. cabozantinib, tivozanib, sunitinib or pazopanib, ipilimumab/nivolumab, pembrolizumab/ axitinib, cabozantinib/nivolumab, axitinib/pembrolizumab, axitinib/avelumab, pembrolizumab/lenvatinib), the treatment landscape is rapidly shifting, paving the way for optimal management in subsequent lines of therapy. Conversely, the rapidness of emerging therapies with the advent of novel agents in the pipeline is adding further complexity to the already overwhelming mRCC landscape. The precise treatment selection remains a debated issue in the absence of head-to-head comparison among the randomized trials. This complexity reinforces the need for optimal therapeutic sequencing, patient selection, and the implication of prognostic risk models for both initial management and systemic therapy paradigms. The quest for optimizing sequence strategies that deliver robust survival, safety, while preserving the quality of life and the ability to tailor therapy to the individual patient remains. An equally important aspect to consider is that the identification of better biomarkers for response to ICIs and TKIs before individualizing therapies corresponding to tailored personalized treatments in mRCC paradigm. Future studies will explore another novel ICI/TKI, TKI, HIF-2a, and combinatorial therapies galvanized with personalized treatment approaches to deliver promising and meaningful therapeutic management in patients with advanced and metastatic RCC.

\section{REFERENCES}

1. Bray F, Ferlay J, Soerjomataram I, Siegel RL, Torre LA, Jemal A. Global cancer statistics 2018: GLOBOCAN estimates of incidence and mortality worldwide for 36 cancers in 185 countries. CA Cancer J Clin. Nov 2018;68(6):394-424. doi:10.3322/caac. 21492

$2 . \quad C z a r n e c k a$ AM, Kornakiewicz A, Kukwa W, Szczylik C. Frontiers in clinical and molecular diagnostics and staging of mccRCC. Future Oncol. May 2014;10(6):1095-111. doi:10.2217/fon.13.258 3. Wang J, Li X, Wu X, et al. Role of immune checkpoint inhibitor-based therapies for $\mathrm{mRCC}$ in the first-line setting: A Bayesian net work analysis. EBioMedicine. Sep 2019;47:78-88 doi:10.1016/j.ebiom.2019.08.006

4. Fyfe G, Fisher RI, Rosenberg SA, Sznol M, Parkinson DR, Louie AC. Results of treatment of 255 patients with mRCC who received highdose recombinant interleukin-2 therapy. J Clin Oncol. Mar 1995;13(3):688-96. doi:10.1200/ JCO.1995.13.3.688

5. McDermott DF, Regan MM, Clark JI, et al. Randomized phase III trial of high-dose interleukin-2 versus subcutaneous interleukin-2 and interferon in patients with mRCC. J Clin Oncol. Jan 1 2005;23(1):133-41. doi:10.1200/ JCO.2005.03.206

6 Jonasch E. Implications of VHL-HIF pathway dysregulation in renal cell carcinoma: current therapeutic strategies and challenges. Kidney Cancer Journal. 2020;18(1):6-10.

7. Motzer RJ, Hutson TE, Tomczak P, et al. Overall survival and updated results for sunitinib compared with interferon alfa in patients with mRCC. J Clin Oncol. Aug 1 2009;27(22):3584-90. doi:10.1200/JCO.2008.20.1293

8. Escudier B, Eisen T, Stadler WM, et al. Sorafenib in advanced clear-cell renal-cell carcinoma. N Engl J Med. Jan 11 2007;356(2):125-34 doi:10.1056/NEJMoao6o655

9. Motzer RJ, Hutson TE, Cella D, et al Pazopanib versus sunitinib in metastatic renal-cell carcinoma. NEnglJ Med.Aug 22 2013;369(8):72231. doi:10.1056/NEJMoa1303989

10. Escudier B, Bellmunt J, Negrier S, et al. Phase III trial of bevacizumab plus interferon alfa-2a in patients with mRCC (AVOREN) final analysis of overall survival. J Clin Oncol. May 1 2010;28(13):2144-50. doi:10.1200/ JCO.2009.26.7849

11. Mehta A, Sonpavde G, Escudier B. Tivozanib for the treatment of renal cell carcinoma: results and implications of the TIVOtrial. Future Oncol. Aug 2014;10(11):1819-26. doi:10.2217/fon.14.120

12. Choueiri TK, Halabi S, Sanford BL et al. Cabozantinib Versus Sunitinib As Initial Targeted Therapy for Patients With mRCC of Poor or Intermediate Risk: The Alliance Ao31203 CABOSUN Trial. J Clin Oncol. Feb 20 2017;35(6):591-597. doi:10.1200/ JCO.2016.70.7398

13. Hudes G, Carducci M, Tomczak P, et al. Temsirolimus, interferon alfa, or both for aRCC. N Engl J Med. May 31 2007;356(22):2271-81. doi:10.1056/NEJMoao66838
14. Tannir NM, Jonasch E, Albiges Prospective Evaluation in mNCRCC (ESPN): A Randomized Multicenter Phase 2 Trial. Eur Urol. May 2016;69(5):866-74. doi:10.1016/j. eururo.2015.10.049

15. Motzer RJ, Tannir NM, McDermott DF, et al. Nivolumab plus Ipilimumab versus Sunitinib in aRCC. N Engl J Med. Apr 5 2018;378(14):12771290. doi:10.1056/NEJMoa1712126

16. Rini BI, Plimack ER, Stus V, et al. Pembrolizumab plus Axitinib versus Sunitinib for aRCC. N Engl J Med. Mar 21 2019;380(12):11161127. doi:10.1056/NEJMoa1816714

17. Hasanov E, Jonasch E. MK-6482 as a potential treatment for von Hippel-Lindau disease-associated ccRCC. Expert Opin Investig Drugs. May 2021;30(5):495-504. doi:10.1080/13 543784.2021 .1925248

18. Kwilas AR, Donahue RN, Tsang KY, Hodge JW. Immune consequences of tyrosine kinase inhibitors that synergize with cancer immunotherapy. Cancer Cell Microenviron. 2015;2(1) doi:10.1480o/ccm.677

19. Rini BI, Powles T, Atkins MB, et al Atezolizumab plus bevacizumab versus sunitinib in patients with previously untreated metastatic renal cell carcinoma (IMmotion151): a multicentre, open-label, phase 3 , randomised controlled trial. Lancet. Jun 15 2019;393(10189):2404-2415. doi:10.1016/So140-6736(19)30723-8

20. Motzer RJ, Penkov K, Haanen J, et al. Avelumab plus Axitinib versus Sunitinib for aRCC. N Engl J Med. Mar 21 2019;380(12):11031115. doi:10.1056/NEJMoa1816047

21. Albiges L, Barthelemy P, Gross-Goupil M, Negrier S, Needle MN, Escudier B. TiNivo: safety and efficacy of tivozanib-nivolumab combination therapy in patients with mRCC. Ann Oncol. Jan 2021;32(1):97-102. doi:10.1016/j. annonc.2020.09.021

22. Amin A, Plimack ER, Ernstoff MS et al. Safety and efficacy of nivolumab in combination with sunitinib or pazopanib in aRCC: the CheckMate 016 study. J Immunother Cancer. Oct 22 2018;6(1):109. doi:10.1186/ S40425-018-0420-0

23. Hamieh L, Beck RL, LeVH, Hsieh JJ.The Efficacy of Lenvatinib Plus Everolimus in Patients with mRCC Exhibiting Primary Resistance to Front-Line Targeted Therapy or Immunotherapy. Clin Genitourin Cancer. Aug 2020;18(4):252-257 e2. doi:10.1016/j.clgc.2020.03.003

24. Motzer RJ, Escudier B, McDermott $\mathrm{DF}$, et al. Nivolumab versus Everolimus in Advanced Renal-Cell Carcinoma. N Engl J Med. Nov 5 2015;373(19):1803-13. doi:10.1056/ NEJMoa1510665

25. Rini BI, Pal SK, Escudier BJ, et al. Tivozanib versus sorafenib in patients with advanced renal cell carcinoma (TIVO-3): a phase 3 , multicentre, randomised, controlled, open-label study. Lancet Oncol. Jan 2020;21(1):95-104 doi:10.1016/S1470-2045(19)30735-1

26. Bono P, Oudard S, Bodrogi I, et al Outcomes in Patients With Metastatic Renal Cell Carcinoma Who Develop Everolimus-Related Hyperglycemia and Hypercholesterolemia: Combined Subgroup Analyses of the RECORD-1 and REACT Trials. Clin Genitourin Cancer. Oct 2016;14(5):406-414. doi:10.1016/j. clgc.2016.04.011

27. Lee $\mathrm{CH}$, Shah AY, Rasco D, et al. Lenvatinib plus pembrolizumab in patients with either treatment-naive or previously treated metastatic renal cell carcinoma (Study 111/ KEYNOTE-146): a phase $1 \mathrm{~b} / 2$ study. Lancet Oncol. Jul 2021;22(7):946-958. doi:10.1016/ S1470-2045(21)00241-2

28. Rini BI, Escudier B, Tomczak P, et al. Comparative effectiveness of axitinib versus sorafenib in advanced renal cell carcinoma (AXIS): a randomised phase 3 trial. Lancet. Dec 3 2011;378(9807):1931-9. doi:10.1016/ So140-6736(11)61613-9 